\title{
Kompetenznetz Public Health zu COVID-19
}

Das „Kompetenznetz Public Health zu COVID-19“ ist ein Ad hoc-Zusammenschluss mehrerer wissenschaftlicher Fachgesellschaften und Verbände aus dem Bereich Public Health, die somit ihre methodische, epidemiologische, statistische, sozialwissenschaftliche und (bevölkerungs-) medizinische Fachkenntnis bündeln. Wissenschaftler aus Deutschland, Österreich und der Schweiz arbeiten daran, schnell interdisziplinäre Expertise zu COVID-19 für die aktuelle Diskussion und Entscheidungsfindung zur Verfügung zu stellen. Dafür werden wissenschaftliche Erkenntnisse zusammengestellt, aufbereitet und in möglichst leicht verständlicher Form verbreitet.

Die AG „Indirekte Gesundheitsfolgen von Maßnahmen des Infektionsschutzes “ hat ein erstes Hintergrundpapier verfasst: Indirekte gesundheitliche Wirkungen von Maßnahmen zum Infektionsschutz können entstehen, wenn sich in der Folge soziale und wirtschaftliche Rahmenbedingungen so verändern, dass gesundheitliche Risiken wie Armut, Arbeitslosigkeit, prekäre Arbeitsbedingungen, psychische Belastungen, eingeschränkte medizinische Versorgung und Bildung, soziale Ungleichheit und Umwelt- 
belastungen mittel- und langfristig zunehmen. Um hier frühzeitig und parallel zu den derzeit akuten Reaktionen auf die COVID19 Pandemie gegensteuern zu können, ist eine Einschätzung möglicher indirekter Folgen auf Basis verfügbaren Wissens hilfreich.

In der AG „Übergangsstrategien“ wurde die aktuelle wissenschaftliche Erkenntnislage zu erwünschten wie unerwünschten Effekten von temporären Schulschließungen und Wiedereröffnungen zusammengestellt. Dabei musste bis auf wenige Ausnahmen auf Studien zurückgegriffen werden, die das Thema während früherer viraler Epidemien untersucht haben. Das Fazit: Schulschließungen haben begrenzte Effekte und erkennbare Risiken, bei Wiedereröffnungen macht es Sinn, Schutzmaßnahmen in Schulen und auf Schulwegen umzusetzen.

Die AG „Gesundheitliche Aspekte der sozialen Isolation “ verfasste ein Fact Sheet zum Thema ältere Menschen am Arbeitsplatz. Das Alter stellt eine wesentliche und unabwendbare Eigenschaft eines Menschen dar. Ein selektives Fernhalten älterer Beschäftigter vom Arbeitsplatz kann zu sozialer Isolation und damit zu einer Risikoerhöhung insbesondere für psychische Erkrankungen, zu Arbeitsplatzunsicherheit und Arbeitslosigkeit führen. Auch angesichts des vergleichsweise geringen - wenngleich nach aktuellem Erkenntnisstand vorhandenen - rein altersbezogenen Risikos ist ein selektives Fernhalten älterer Beschäftigter vom Arbeitsplatz zu vermeiden.

Die AG „Gesundheit und Arbeit“ verfasste ein Fact Sheet zu Beschäftigten mit erhöhtem Krankheitsrisiko. Berufstätige Personen, die erhöhtes Risiko tragen für einen schweren Covid-19-Krankheitsverlauf bzw. Tod, d. h. insbesondere ältere Personen mit bestimmten chronischen Erkrankungen, sollten beruflich nicht in Tätigkeiten mit einem erhöhten Ansteckungsrisiko eingesetzt werden. Ist dies weder am regulären Arbeitsplatz, ggf. durch technische, organisatorische oder personenbezogene Schutzmaßnahmen, durch innerbetriebliche Umsetzung, noch durch Telearbeit/Homeoffice realisierbar, empfehlen wir eine bezahlte Freistellung. Diese könnte, in Analogie zur Entgeltfortzahlung bei Mutterschaft und Beschäftigungsverbot (U2), von Krankenkassen und Unfallkassen finanziert werden. Ob beruflich - über das Risiko in der Allgemeinbevölkerung hinausgehend - ein erhöhtes Ansteckungsrisiko besteht, ergibt die Gefährdungsbeurteilung. Die Beurteilung des individuell erhöhten Krankheitsrisikos sollte ärztlicherseits gemeinsam mit der betroffenen Person erfolgen. Wo betriebsärztliche Betreuung gewährleistet ist, ließe sich das, entsprechend der ArbMedVV (Teil 2), über eine Angebotsvorsorge bewerkstelligen. Anderenfalls obläge die Aufgabe, das Krankheitsrisiko zu beurteilen, den behandelten Ärzt/innen.

Die AG „Gesundheitliche Aspekte der sozialen Isolation“ verfasste ein Policy Brief mit dem Titel: „Psychosoziale Folgen von Isolations und Quarantänemaßnahmen: Womit müssen wir rechnen? Was können wir dagegen tun?" Isolations- und Quarantänemaßnahmen haben belegbare negative Folgen für die psychosoziale Gesundheit; dazu zählen erhöhte Depressivität, Ängstlichkeit, posttraumatische Belastung, Wut, Stresserleben und Einsamkeit. Darüber hinaus wurden Stigmatisierungserfahrungen berichtet. Bekannte Risikogruppen sind Menschen mit psychischen Vorerkrankungen und Mitarbeiterinnen und Mitarbeiter im Gesundheitswesen. Auch Angehörige von vulnerablen und belasteten Personengruppen müssen berücksichtigt werden.

Die AG „Wirksamkeit und Nebenwirkungen von Nicht-Pharmakologischen Interventionen “ verfasste ein Fact Sheet zum Nutzen von Gesichtsmasken. Basierend auf aktuellen Studienergebnissen ist der Nutzen von Gesichtsmasken in der Öffentlichkeit weiter unklar. Es ist weiterhin strengstens auf das Einhalten der Abstandsregeln und der Händehygiene zu achten. Die Maskenpflicht darf weder als Anlass noch als Begründung für weitere Lockerungen anderer Maßnahmen dienen.

Alle Ergebnisse und Paper des Kompetenznetzes Public Health zu COVID-19 sind abrufbar unter: https://www.public-healthcovid19.de/de/produkte.html

Nach Angaben des Kompetenznetzes Public Health zu COVID-19 